\title{
Testicular Cancer cTX TNM Finding v8
}

National Cancer Institute

\section{Source}

National Cancer Institute. Testicular Cancer CTX TNM Finding v8. NCI Thesaurus. Code C140194.

Testicular cancer in which the primary tumor cannot be assessed. (from AJCC 8th Ed.) 